\title{
Oscillating Thrombi in Bilateral Extracranial Internal Carotid Arteries Demonstrated on Ultrasonography: Two Autopsy Cases of Cardioembolic Stroke
}

\author{
Koji Tanaka ${ }^{1}$, Toshiyuki Uehara ${ }^{1}$, Masahiro Miyoshi ${ }^{1}$, Fumio Miyashita ${ }^{1}$, \\ Taka-aki Matsuyama ${ }^{2}$, Hatsue Ishibashi-Ueda ${ }^{2}$ and Kazunori Toyoda ${ }^{1}$
}

\begin{abstract}
We herein report two autopsy cases of severe cardioembolic stroke with oscillating thrombi in the bilateral extracranial internal carotid arteries (ICAs) demonstrated on carotid ultrasonography performed on admission. An autopsy study of Case 1 conducted on the third hospital day revealed no thrombi, while that of Case 2 conducted on the 42nd hospital day revealed red thrombi in the extracranial ICAs. Our postmortem studies confirm that oscillating thrombi may be seen in the region of blood stasis caused by occlusion of the distal portion of the ICA, thus reflecting a pre-state of thrombus formation.
\end{abstract}

Key words: ischemic stroke, atrial fibrillation, oscillating thrombus, internal carotid artery occlusion, pathology, carotid ultrasonography

(Intern Med 52: 1243-1247, 2013)

(DOI: 10.2169/internalmedicine.52.9558)

\section{Introduction}

In patients with acute ischemic stroke, making an early and accurate assessment of the carotid or cerebral arteries is important. Neurosonographic studies, including carotid ultrasonography, are real-time, noninvasive techniques that can be followed up easily. An oscillating intraluminal mass originating from the internal carotid artery (ICA) on B-mode ultrasonography, the lumen of which was filled and oscillated with the cardiac cycle, in an acute cardioembolic stroke patient was first reported by Yonemura et al. (1). This condition was subsequently named "oscillating thrombus" as a specific finding of acute embolic occlusion of the ICA (2). We herein report two autopsy cases of acute ischemic stroke with atrial fibrillation (AF) involving patients with oscillating thrombi in the bilateral extracranial ICAs detected on carotid ultrasonography.

\section{Case Reports}

\section{Case 1}

A 90-year-old woman with chronic $\mathrm{AF}$ was transported to our hospital by ambulance due to a sudden disturbance of consciousness. A neurological examination performed on admission showed severe consciousness impairment, tetraparesis, decorticate rigidity and bilateral Babinski signs. The oculocephalic reflex was intact. Although the patient was taking warfarin, her prothrombin time-international normalized ratio (PT-INR) was 0.98. No early ischemic signs were detected on head CT; however, an extended high-intensity area was found in the bilateral cerebral hemispheres on a diffusion-weighted image (DWI) of magnetic resonance imaging (MRI) (Fig. 1A). The bilateral ICAs were not present on magnetic resonance angiography (MRA) (Fig. 1B). On B-mode carotid ultrasonography, oscillating thrombi were seen in the bilateral origins of the ICA (Fig. 1C). Doppler ultrasonography showed the disappearance of end-diastolic

${ }^{1}$ Department of Cerebrovascular Medicine, National Cerebral and Cardiovascular Center, Japan and ${ }^{2}$ Department of Pathology, National Cerebral and Cardiovascular Center, Japan

Received for publication December 19, 2012; Accepted for publication January 29, 2013

Correspondence to Dr. Toshiyuki Uehara, tuehara@ncvc.go.jp 


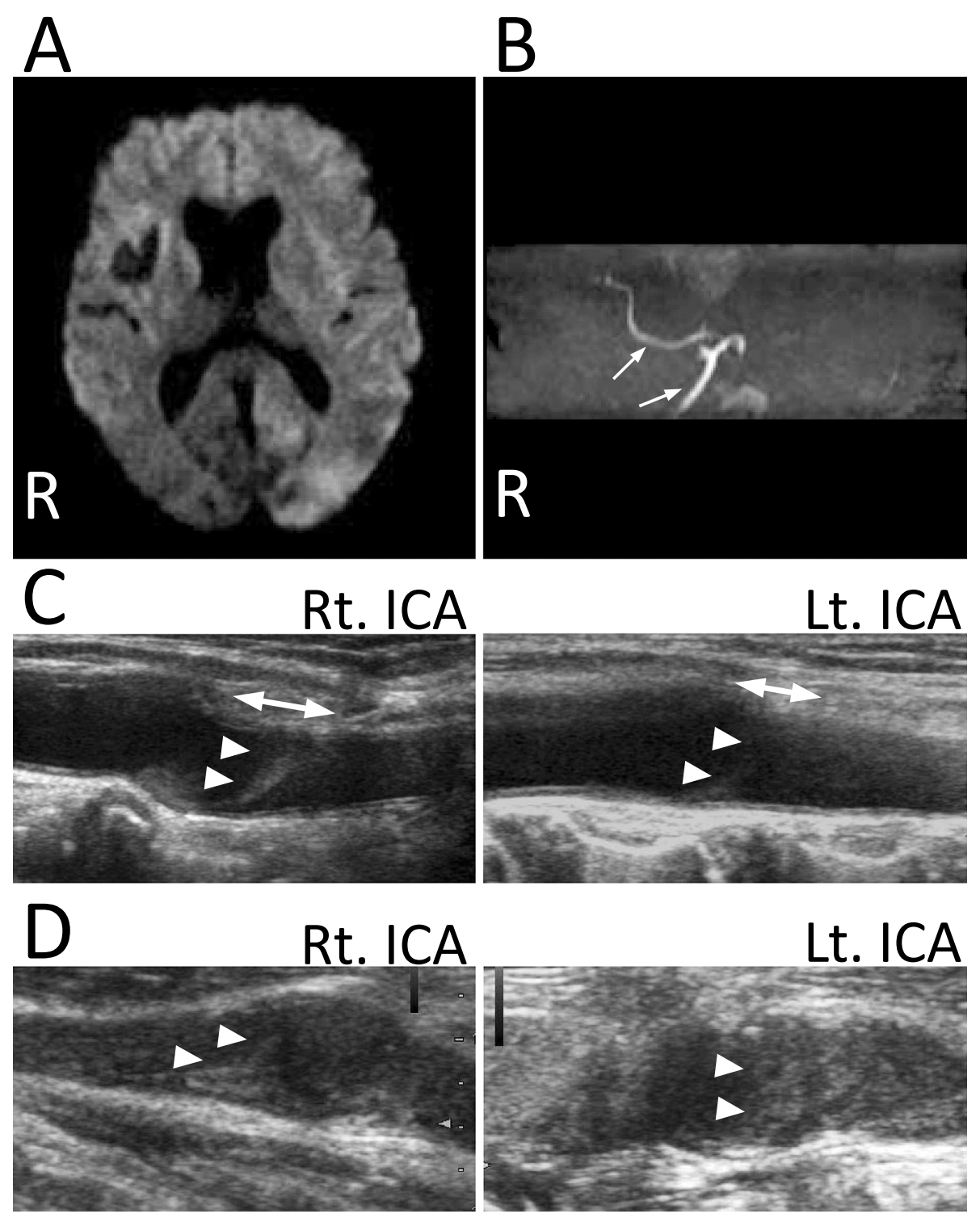

Figure 1. MRI and ultrasonography findings in Case 1 (A-C) and Case 2 (D). In Case 1 on admission, extended high-intensity lesions were seen in the bilateral hemispheres excluding the right posterior cerebral artery (PCA) territory on a diffusion-weighted image ( $T R=0 \mathrm{msec}, \mathrm{TE}=100 \mathrm{msec}$, $b=1,000$ ) (A). On MRA, the basilar artery and the right PCA (arrows) were visible; however, the anterior circulation and the left PCA were not (AP view) (B). B-mode carotid ultrasound showed thrombi arising from the bilateral origins of the internal carotid arteries (ICAs) (two small arrowheads) and oscillating with the cardiac cycle (between the ranges of the double-headed arrows) (C). In Case 2 on the fifth day, B-mode carotid ultrasound demonstrated thrombi with increased echo intensity and decreased mobility (D).

flow velocities in the bilateral common carotid arteries and no flow signals in the bilateral ICAs. The patient's brain stem reflex disappeared on the second day, and she died of respiratory arrest on the third day. A postmortem study was performed 2.5 hours after death. An autopsy revealed diffuse edema of the brain with central herniation. The vessels, including the bilateral anterior cerebral arteries, bilateral middle cerebral arteries and left posterior cerebral artery, were occluded with red thrombi (Fig. 2A). Although the bilateral intracranial ICAs were occluded, there were no thrombi in the bilateral extracranial ICAs (Fig. 2B). Infarcts were also seen in the right kidney. An intramural thrombus was found in the left atrial appendage (LAA) (Fig. 2C).

\section{Case 2}

A 79-year-old woman with chronic AF was admitted due to a disturbance of consciousness. On admission, she displayed decerebrate rigidity; however, her oculocephalic reflex was intact. Although she had been prescribed warfarin, her PT-INR was 1.04. DWI revealed high-intensity areas in the bilateral cerebral hemispheres. The bilateral ICAs were not present on MRA. On B-mode carotid ultrasonography, 


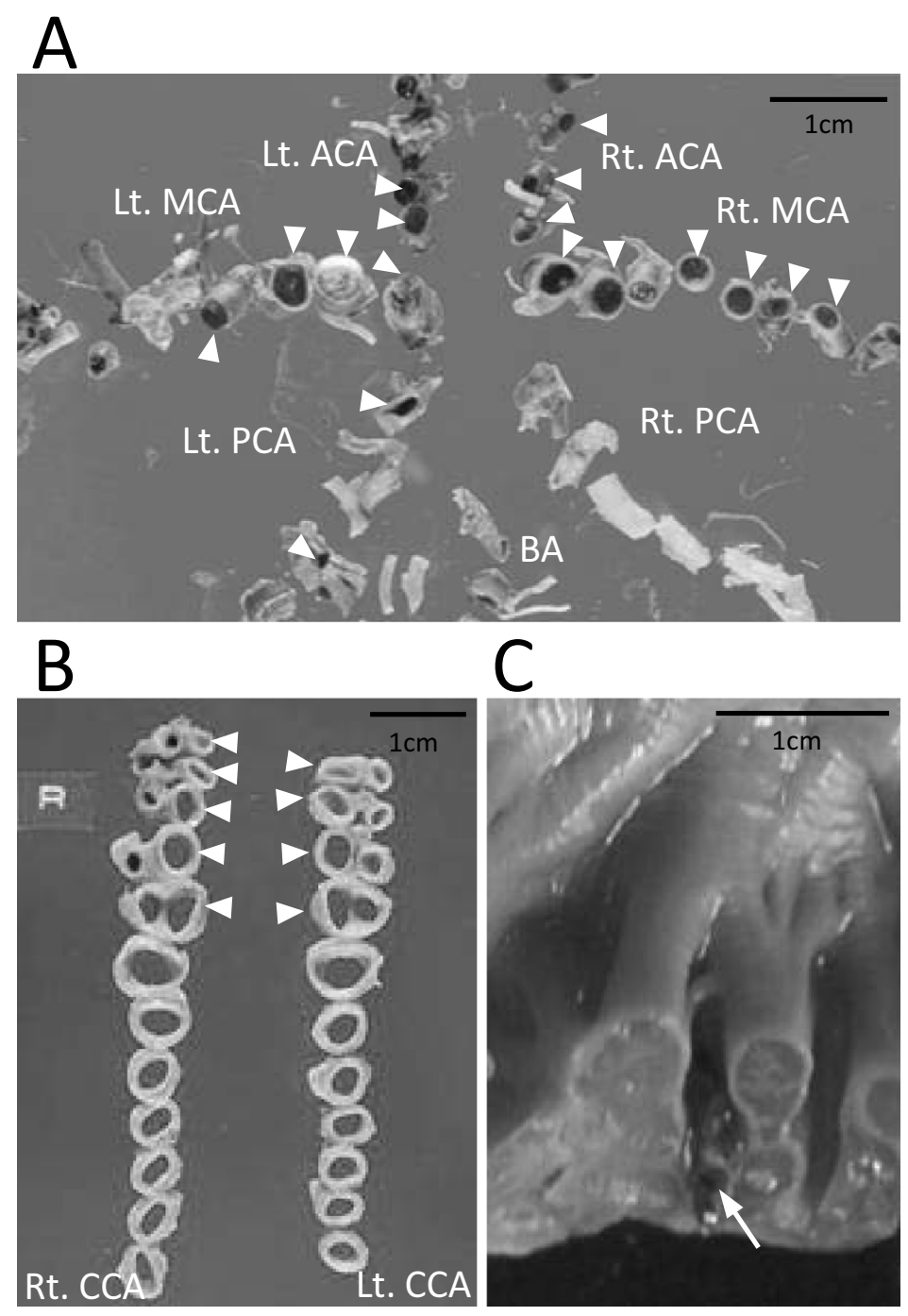

Figure 2. Pathological findings in Case 1. The intracranial ICAs, bilateral anterior cerebral arteries, bilateral middle cerebral arteries and left PCA were occluded with red thrombi (A). There were no thrombi in the bilateral extracranial ICAs (B). An intraluminal thrombus was seen in the left atrial appendage (LAA) (C).

oscillating thrombi were seen in the bilateral origins of the ICA. On the day of admission, she developed respiratory dysfunction. Chest $\mathrm{X}$-rays revealed congestion of the lungs. Based on the electrocardiography and cardiac ultrasound findings, she was diagnosed with Takotsubo cardiomyopathy. She was intubated and placed on mechanical ventilation. On the third day, her brain stem reflex and spontaneous breathing disappeared. Carotid ultrasonography performed on the fifth day revealed oscillating thrombi with increased echo intensity and decreased mobility (Fig. 1D). She died of septic shock 42 days after onset. A postmortem study was performed 5.5 hours after death, which revealed diffuse softening of the brain and occlusion of the bilateral ICAs with fresh red thrombi (Fig. 3A, C). The right ECA was also occluded with a pathologically proven fibrin-rich thrombus (Fig. 3D). Atherosclerotic changes in the bilateral extracranial ICAs were mild. An examination of the heart revealed global subendocardial coagulative myocyte necrosis compatible with Takotsubo cardiomyopathy. A red thrombus measuring $2 \mathrm{~cm}$ in diameter was seen in the LAA (Fig. 3B).

\section{Discussion}

Kimura et al. (2) reported an autopsy case of an oscillating thrombus in the unilateral extracranial ICA detected on admission. In their postmortem study performed 24 days after onset, the lumen of the ICA was obstructed with a fresh string-like thrombus. Similarly, our autopsy study of Case 2 conducted 42 days after onset showed red thrombi obstructing the lumens of the ICAs. The right extracranial ECA was occluded with a fibrin-rich organized thrombus, thus suggesting another cardiogenic thrombus had occluded the right ECA after admission.

A spontaneous contrast or smoke-like echo is usually found as an echocardiographic finding in patients in a state of blood stasis. This finding is related to the interaction of red blood cells and plasma proteins and to erythrocyte rouleaux formation in low-flow and low-shear-rate condi- 


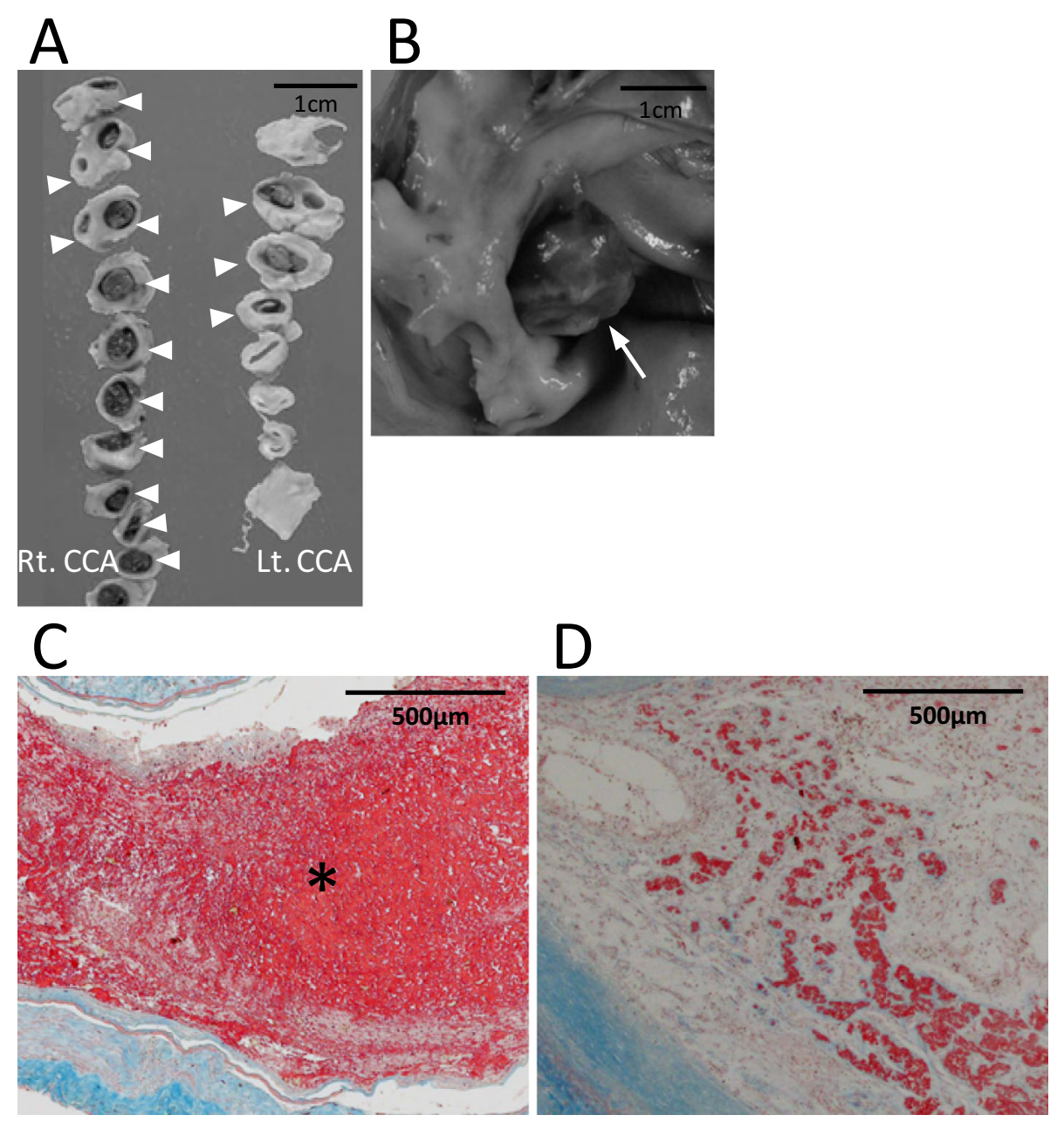

Figure 3. Pathological findings in Case 2. The bilateral extracranial ICAs, right external carotid artery (ECA) and right common carotid artery were occluded (A). A red thrombus measuring $\mathbf{2} \mathrm{cm}$ in diameter was seen in the LAA (B). Pathologically, the thrombus in the right ICA was a fresh red thrombus (*), while that in the right ECA was a fibrin-rich thrombus $(C, D)$ (Masson's trichrome staining).

tions $(3,4)$. Such a finding is considered to indicate a prestate of thrombus formation. Oscillating thrombi may also be seen in the area of blood stasis caused by occlusion of the distal portion of the ICA. Our two postmortem studies indicate that oscillating thrombi cannot always be pathologically detected in the acute phase and that propagating fresh red thrombi are formed with the passage of time. Neurological deficits caused by bilateral ICA occlusions vary greatly $(5,6)$. Cases of bilateral ICA occlusions with severe bilateral neurological symptoms and consciousness disorders that mimic brain stem infarctions were first described by Fisher (5) in 1954. Most of the reported patients were thought to have developed occlusions at different time points. Simultaneous occlusion of the bilateral ICAs appears to be relatively rare and it is reportedly caused by head trauma (7-10), catheter manipulation (11) and cardiac embolism (12-15). In our cases, the bilateral ICAs were thought to be simultaneously occluded because oscillating thrombi were seen in the bilateral ICAs on admission. A sudden onset of bilateral ICA occlusion leads to a very poor prognosis associated with poor collateral circulation. Because patients with $\mathrm{AF}$ are at risk of severe stroke, administering appropriate anticoagulation therapy is therefore necessary.

The authors state that they have no Conflict of Interest (COI).

This work was supported by JSPS KAKENHI Grant Number 24591309.

\section{References}

1. Yonemura K, Kimura K, Yonemitsu M, Hashimoto Y, Uchino M. The intravascular mobile structure by duplex carotid ultrasonography in cardioembolic internal carotid artery occlusion. Rinsho Shinkeigaku 36: 1125-1128, 1996 (in Japanese, Abstract in English).

2. Kimura K, Yasaka M, Minematsu K, et al. Oscillating thromboemboli within the extracranial internal carotid artery demonstrated by ultrasonography in patients with acute cardioembolic stroke. Ultrasound Med Biol 24: 1121-1124, 1998.

3. Wang XF, Liu L, Cheng TO, Li ZA, Deng YB, Wang JE. The relationship between intracardiovascular smoke-like echo and eryth- 
rocyte rouleaux formation. Am Heart J 124: 961-965, 1992.

4. Merino A, Hauptman P, Badimon L, et al. Echocardiographic "smoke" is produced by an interaction of erythrocytes and plasma proteins modulated by shear forces. J Am Coll Cardiol 20: 16611668, 1992.

5. Fisher M. Occlusion of the carotid arteries: further experiences. AMA Arch Neurol Psychiatry 72: 187-204, 1954.

6. Field WS, Edwards WH, Crawford ES. Bilateral carotid artery thrombosis. Arch Neurol 4: 369-383, 1961.

7. Ojemann RG, Moser HW. Acute bilateral internal carotid artery occlusion; report of a case following a parachute jump. Neurology 14: 565-568, 1964.

8. Yashon D, Johnson AB 2nd, Jane JA. Bilateral internal carotid artery occlusion secondary to closed head injuries. J Neurol Neurosurg Psychiatry 27: 547-552, 1964.

9. Robinson RG, Gwynne JF. Bilateral internal carotid artery thrombosis after closed head injury. Acta Neurochir (Wien) 44: 137-142, 1978.
10. Scherman BM, Tucker WS. Bilateral traumatic thrombosis of the internal carotid arteries in the neck: a case report with review of the literature. Neurosurgery 10: 751-753, 1982.

11. Hussain SA, Bayar A. Catheter-induced bilateral internal carotid artery occlusion. J Vasc Surg 20: 1000-1003, 1994.

12. Yamaguchi S, Oki S, Ogasawara $\mathrm{H}$, Sato $\mathrm{H}$, Ito $\mathrm{Y}$, Hibino S. A case of simultaneous bilateral carotid occlusion. No Shinkei Geka 25: 247-251, 1997 (in Japanese).

13. Kawano H, Suga T, Terasaki $T$, et al. Decerebrate rigidity after bilateral carotid arteries occlusion. Rinsho Shinkeigaku 44: 545-548, 2004 (in Japanese, Abstract in English).

14. Hagiwara N, Toyoda K, Fujimoto S, Okada Y. Extensive bihemispheric ischemia caused by acute occlusion of three major arteries to the brain. J Neurol Sci 212: 99-101, 2003.

15. Kwon SU, Lee SH, Kim JS. Sudden coma from acute bilateral internal carotid artery territory infarction. Neurology 58: 1846-1849, 2002.

(C) 2013 The Japanese Society of Internal Medicine http://www.naika.or.jp/imonline/index.html 